contrast with the study involving the dorsal forearm where the mean difference value, i.e., DL, for the four Ss tested was $6 \mathrm{~mm}$.

In summary, it has been shown that the DL for supraliminal two-point tactual stimuli applied to the back is greater than a comparable DL derived from tactual stimuli applied to the dorsal forearm. This is consistent with the fact that the two-point threshold is considerably greater for the back than for the dorsal forearm.
REFERENCES

CROSS, H. A., BOYER, W. N., \& GUYOT, G. W Determination of a DL using two-point tactual stimuli: A signal-detection approach. Psychonomic Science, 1970, 00, 000-000.

GREEN, D. M., \& SWETS, J. A. Signal detection theory and psychophysics. New York: Wiley 1966.

HILGARD, E. R. Introduction to psychology, New York: Harcourt, Brace, \& World, 1953.

UNDERWOOD, B. J. Experimental psychology. New York: Appleton-Century-Crofts, 1966.

\title{
Magnitude of model's apparent pain and ability to aid the model as determinants of observer reaction time*
}

\author{
ROBERT A. BARON $\dagger$ \\ University of South Carolina, Columbia, S.C. 29208
}

Thirty-two undergraduate females participated in an experiment designed to investigate the effects of magnitude of apparent suffering of a live peer model and the instructions concerning their ability to aid this individual on 0 reaction time (RT). It was predicted that under conditions where Os were informed that they could influence the suffering of the model by the speed of their responses, RT would decrease as the magnitude of pain evidenced by this individual increased. However, under conditions where Os were informed that they could not aid the model, it was expected that RT would actually increase as the degree of pain shown by this person increased. Results supported these predictions.

A number of recent experiments (e.g., Bandura \& Rosenthal, 1966; Berger, 1962; Craig \& Lowery, 1969) have demonstrated that exposure to the apparent pain and suffering of another person may elicit signs of physiological arousal among Os. An additional study (Di Lollo \& Berger, 1965) has reported that such experience may also produce significant decrements in $O$ reaction time (RT). Thus, it appears that apparent pain in another may influence both the physiological reactions and the overt behavior of Os.

A recent experiment (Baron, 1970) sought to extend the findings of the Di Lollo and Berger study by investigating the effects of three levels of apparent suffering on the part of a live peer model on O RT. In accordance with the findings reported by these authors and certain suggestions made by Berger (1962, p. 463),

*The author wishes to express his appreciation to Rollie O. Waters and Larry M. Cope for their assistance in the collection of the data, and to Murray $O$. Meetze for his aid in the construction of the experimental apparatus.

†Requests for reprints should be sent to the author at the Department of Psychology, University of South Carolina, Columbia, S.C. 29208.

it was hypothesized that $\mathrm{O}$ RT would decrease as the magnitude of pain cues emitted by the model increased. However, contrary to this prediction, it was found that $O$ RT actually increased significantly as the intensity of pain evidenced by the model increased.

The present study was designed to examine the possibility that the conflicting findings of the Di Lollo \& Berger (1965) and Baron (1970) experiments arose from certain procedural differences which led Os in the two studies to assume different degrees of control over the suffering of the model. In the Di Lollo and Berger study, conditions were arranged so that there was often a close temporal contiguity between $O s^{\prime}$ overt responses and the cessation of shocks to the model. As a result of this association, many Os may have assumed that they could influence the duration of the apparent shocks to the model. Thus, the decrements in RT that were observed may have been produced by their subsequent attempts to "aid" this individual.

In the Baron (1970) study, on the other hand, such a close temporal contiguity between $\mathrm{Os}^{\mathrm{s}}$ overt responses and cessation of shock to the model was lacking. Thus, it seems unlikely that Os in this study concluded that they could affect the suffering of this individual by varying the speed of their responses. Instead, they were faced with a situation in which they could observe the suffering of another individual but could not aid him in any manner. In order to reduce the aversiveness of this situation, they may have engaged in various self-generated competing responses, such as looking away from the model or focusing their attention on irrelevant stimuli in the experimental room. Such activities, which have been reported in previous studies (e.g., Bandura \& Rosenthal, 1966; Berger, 1962), may then have interfered with Os' RT by reducing their attention to the experimental task.

It was reasoned that if the above suggestions were accurate, instructions to Os regarding their ability to influence the shocks to the model would exert an important effect upon O RT. Specifically, it was predicted that under conditions where Os were informed that they could control the shocks to the model, O RT would decrease as the magnitude of pain cues emitted by the model increased. However, under conditions where Os were informed that they could not influence the shocks to the model, O RT would actually increase as the magnitude of pain cues emitted by this individual increased.

\section{OBSERVERS}

Thirty-two undergraduate females enrolled in sections of elementary psychology at the University of South Carolina participated in the experiment. Students took part in the study in order to fulfill a course requirement.

\section{APPARATUS}

The apparatus consisted of a Hunter timer (Model 111-C), a Lafayette digital stop clock (Model 5721), two stimulus panels, two telegraph keys, an electric buzzer, and one pair of finger electrodes. One stimulus panel contained red and white signal lights, while the second unit contained only a white light.

\section{DESIGN}

A 2 by 2 factorial design, based upon two levels of apparent shock to the model (low, high) and two levels of instructions regarding the ability of Os to influence the shocks to the model (independent, dependent), was employed. Eight Os were assigned randomly to each cell of this design.

\section{PROCEDURE}

The procedures of the present study were highly similar to those employed in a previous experiment (Baron, 1970). Thus, they will not be described in detail here. Briefly, Os observed a model (a confederate of the $E$ ) receive one of two levels of electric shock, and then responded 


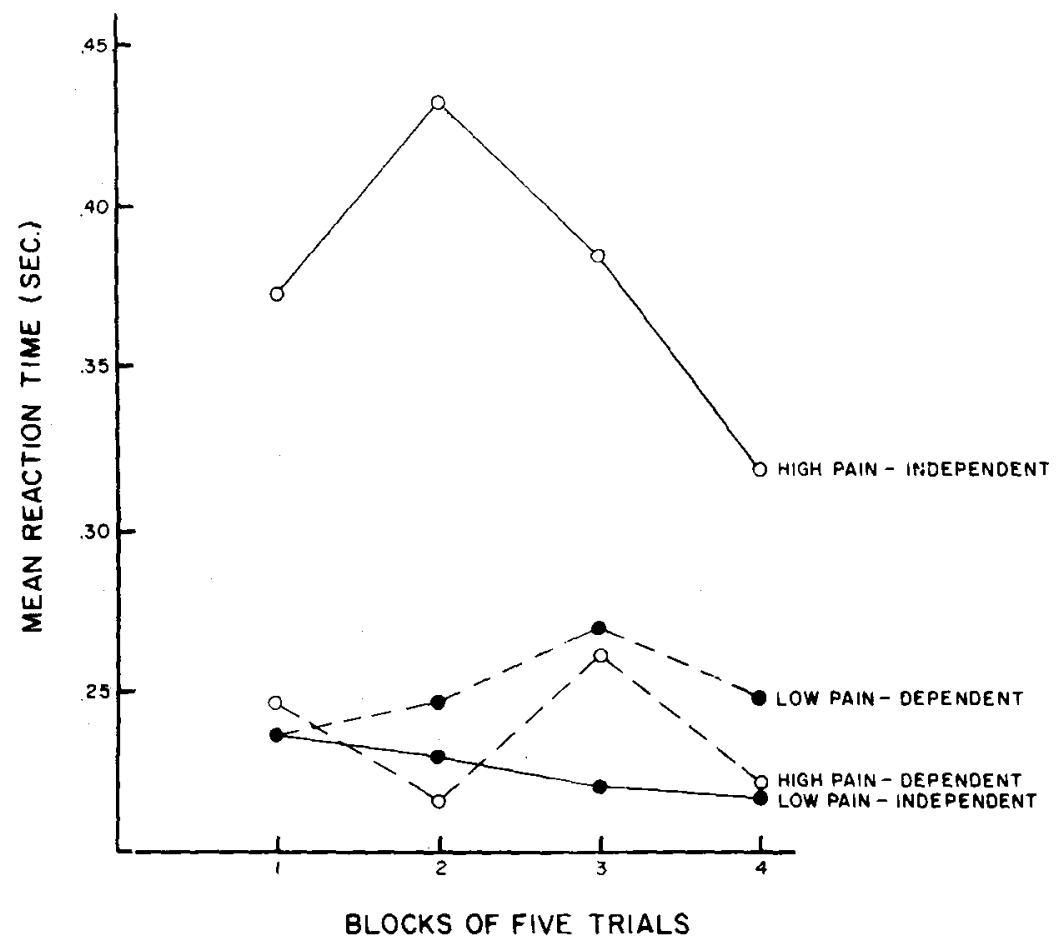

Fig. 1. Mean RTs shown by Ss in four groups as a function of successive blocks of five trials.

in a RT task. In the low-pain condition the $E$ described the shocks to the model as being of low intensity, and the confederate showed no sign of discomfort on occasions when a red light, signaling shock, flashed on. In the high-pain condition the $E$ described the shocks to the model as being of very high intensity. In addition, the confederate jerked his arm sharply, moved in his seat, and groaned as if in pain on occasions when he appeared to receive electric shock. On each trial the $O$ responded in the $\mathrm{RT}$ task only after witnessing the apparent shock to the model and the performance of this individual on the same task.

Os were also provided with explicit instructions concerning the relationship between the speed of their responses and the duration of the shocks to the model. In the independent condition, they were informed that the shocks to the model were introduced automatically by the apparatus and were not under their control. In the dependent condition, Os were informed that the faster they responded on a given trial, the shorter would be the shock to the model on the following trial. Thus, an attempt was made to influence Os' assumptions regarding their ability to aid this individual.

$$
\text { RESULTS }
$$

Figure 1 presents the mean RTs shown by $O s$ in the four groups in each of four successive blocks of five trials. Inspection
RT decreased slightly as magnitude of model's pain cues increased when Os werc informed that the duration of the shocks to this individual depended on the speed of their responses. The failure of the high-pain dependent group to show significant reductions in RT may have resulted from a "floor" effect which prevented any further decrements in RT below that shown by the low-pain dependent group. Future research may reduce the possible occurrence of such effects by employing RT tasks (e.g., the buttonpressing task employed by Di Lollo \& Berger, 1965) in which Os do not show the very short RT obtained in the present study.

The results of the present experiment suggest that the manner in which Os respond to the suffering of another individual may depend upon their perceptions regarding their ability to aid this person. Under conditions where Os were informed that they could not influence the shocks to the model, results suggested that Os engaged in activities designed to "blot out" the suffering of this individual. However, under conditions where they were informed that the duration of the shocks to the model depended upon the speed of their responses, results suggested that $O$ s refrained from such activities and may have engaged in overt attempts to aid this individual. This latter finding suggests that it may be possible to increase the frequency of altruism toward a suffering other by providing conditions that suggest to Os that they can act to reduce or eliminate this suffering. In view of the relatively low incidence of helping behavior reported in several recent experiments concerned with bystander intervention in emergencies (e.g., Latané \& Rodin, 1969), further research designed to investigate these conditions would appear to have important implications.

\section{REFERENCES}

BANDURA, A., \& ROSENTHAL, T. L. Vicarious classical conditioning as a function of arousal level. Journal of Personality \& Social Psychology, 1966, 3, 54-62.

BARON, R. A. Effects of magnitude of model's apparent pain on observer reaction time. Psychonomic Science, 1970, 20, 229-231.

BERGER, S. M. Conditioning through vicarious instigation. Psychological Review, 1962, 69, $450-466$.

CRAIG, K. D., \& LOWERY, H. J. Heart-rate components of conditioned vicarious autonomic responses. Journal of Personality \& Social Psychology, 1969, 11, 381-387.

Di LOLLO, V., \& BERGER, S. M. Effects of apparent pain in others on observer's reaction time. Journal of Personality \& Social Psychology, 1965, 2, 573-575.

LATANÉ, B., \& RODIN, J. A lady in distress: Inhibiting effects of friends and strangers on bystander intervention. Journal of Experimental Social Psychology, 1969. 5. 189-202. 